\title{
Local characterization of algebraic manifolds and characterization of components of the set $S_{f}$
}

\author{
by ZBIGNIEW JELONEK (Kraków)
}

\begin{abstract}
We show that every $n$-dimensional smooth algebraic variety $X$ can be covered by Zariski open subsets $U_{i}$ which are isomorphic to closed smooth hypersurfaces in $\mathbb{C}^{n+1}$.

As an application we show that for every (pure) $n$-1-dimensional $\mathbb{C}$-uniruled variety $X \subset \mathbb{C}^{m}$ there is a generically-finite (even quasi-finite) polynomial mapping $f: \mathbb{C}^{n} \rightarrow \mathbb{C}^{m}$ such that $X \subset S_{f}$.

This gives (together with [3]) a full characterization of irreducible components of the set $S_{f}$ for generically-finite polynomial mappings $f: \mathbb{C}^{n} \rightarrow \mathbb{C}^{m}$.
\end{abstract}

1. Introduction. In Section 2 we prove the following theorem:

Let $X$ be an $n$-dimensional algebraic variety and $x \in X$ be a smooth point on $X$. Then there is a Zariski open neighborhood $U_{x} \subset X$ of $x$ which is isomorphic to a closed smooth hypersurface in $\mathbb{C}^{n+1}$.

In particular it implies that every $n$-dimensional smooth algebraic variety $X$ can be covered by Zariski open subsets $U_{i}$ which are isomorphic to closed smooth hypersurfaces in $\mathbb{C}^{n+1}$. Moreover, we find that every algebraic variety of dimension $n>0$ has infinitely many pairwise non-isomorphic smooth models in $\mathbb{C}^{n+1}$.

As an application of the theorem above we give a characterization of components of the set $S_{f}$ of points at which a polynomial mapping $f$ : $\mathbb{C}^{n} \rightarrow \mathbb{C}^{m}$ is not proper. Let us recall that $f$ is not proper at a point $y$ if there is no neighborhood $U$ of $y$ such that $f^{-1}(\operatorname{cl}(U))$ is compact. In [3] we showed that the set $S_{f}$ (if non-empty) has pure dimension $n-1$ and it is $\mathbb{C}$-uniruled, i.e., for every point $x \in S_{f}$ there is an affine parametric curve through this point. In this paper we show that, conversely, for every $\mathbb{C}$-uniruled $n$-1-dimensional variety $X \subset \mathbb{C}^{m}$ (where $2 \leq n \leq m$ ), there

2000 Mathematics Subject Classification: 14E10, 14E22, 14E40.

Key words and phrases: polynomial mappings, affine space, $\mathbb{C}$-uniruled variety.

This paper is partially supported by a KBN grant no. 2 PO3A 03714. 
is a generically-finite (even quasi-finite) polynomial mapping $F: \mathbb{C}^{n} \rightarrow \mathbb{C}^{m}$ such that $X \subset S_{F}$. This gives (together with [3]) a full characterization of irreducible components of the set $S_{f}$.

2. Zariski open subsets which are affine hypersurfaces. We begin with the following lemma:

Lemma 2.1. Let $X$ be an n-dimensional affine algebraic variety and $w \in X$ be a smooth point on $X$. Then there is a finite, regular birational mapping $\phi: X \rightarrow \mathbb{C}^{n+1}$ such that

(1) $\phi^{-1}(\phi(w))=\{w\}$,

(2) the mapping $d_{w} \phi: T_{w} X \rightarrow \mathbb{C}^{n+1}$ is an embedding.

Proof. We can assume that $X \subset \mathbb{C}^{m}$. Observe that there is a finite projection $f: X \rightarrow \mathbb{C}^{n}$ such that $d_{w} f: T_{w} X \rightarrow \mathbb{C}^{n}$ is an isomorphism. We can assume that $f: X \ni\left(x_{1}, \ldots, x_{n}, x_{n+1}, \ldots, x_{m}\right) \mapsto\left(x_{1}, \ldots, x_{n}\right) \in \mathbb{C}^{n}$. Let $f^{-1}(f(w))=\left\{w_{1}, \ldots, w_{k}\right\}$. By the theorem on the primitive element there is a linear form $z(x)=\sum_{i=n+1}^{m} c_{i} x_{i}$ such that the mapping $\phi:=(f, z)$ is birational. Moreover, we can assume that $z\left(w_{i}\right) \neq z\left(w_{j}\right)$ for $i \neq j$ and consequently $\phi^{-1}(\phi(w))=\{w\}$. This finishes the proof.

REMARK 2.2. In particular, the point $\phi(w)$ is smooth on the variety $\phi(X)$.

For our next step it is convenient to introduce the following special birational mapping $F_{h}$.

Definition 2.3. Let $h \in \mathbb{C}\left[x_{1}, \ldots, x_{n}\right]$ and define $F_{h}: \mathbb{C}^{n+1} \ni\left(x_{1}, \ldots, x_{n}, x_{n+1}\right) \mapsto\left(x_{1}, \ldots, x_{n}, h\left(x_{1}, \ldots, x_{n}\right) x_{n+1}\right) \in \mathbb{C}^{n+1}$.

The mapping $F_{h}$ will be called the $h$-process given by the polynomial $h$. The hypersurface $V_{h}:=\left\{x \in \mathbb{C}^{n+1}: h(x)=0\right\}$ is the vertical hypersurface of the $h$-process $F_{h}$ and the hyperplane $H_{h}:=\left\{x \in \mathbb{C}^{n+1}: x_{n+1}=0\right\}$ is the horizontal hyperplane of $F_{h}$.

Lemma 2.4. The $h$-process $F_{h}$ given by a polynomial $h \in \mathbb{C}\left[x_{1}, \ldots, x_{n}\right]$ is a birational mapping and the restriction $F_{h}: \mathbb{C}^{n+1} \backslash V_{h} \rightarrow \mathbb{C}^{n+1} \backslash V_{h}$ is an isomorphism. Moreover, for every hypersurface

$$
X:=\left\{x \in \mathbb{C}^{n+1}: x_{n+1}^{k}+\sum_{j=1}^{k-1} a_{j}\left(x_{1}, \ldots, x_{n}\right) x_{n+1}^{k-j}=0\right\},
$$

we have $\operatorname{cl}\left(F_{h}(X)\right) \cap V_{h} \subset V_{h} \cap H_{h}$.

Proof. Indeed, it is easy to see that the hypersurface $\operatorname{cl}\left(F_{h}(X)\right)$ has equation $x_{n+1}^{k}+\sum_{j=1}^{k-1} a_{j}\left(x_{1}, \ldots, x_{n}\right) h^{j} x_{n+1}^{k-j}=0$, and the lemma follows.

Our first main result is: 
TheOREM 2.5. Let $X$ be an $n$-dimensional algebraic variety and $x \in X$ be a smooth point on $X$. Then there is a Zariski open neighborhood $U_{x} \subset X$ of $x$ which is isomorphic to a closed smooth hypersurface in $\mathbb{C}^{n+1}$.

Proof. Let $\phi: X \rightarrow \mathbb{C}^{n+1}$ be a birational embedding as in Lemma 2.1. Set $X_{1}:=\phi(X)$. By a change of variable we can assume that

$$
X_{1}=\left\{x \in \mathbb{C}^{n+1}: x_{n+1}^{k}+\sum_{j=1}^{k-1} a_{j}\left(x_{1}, \ldots, x_{n}\right) x_{n+1}^{k-j}=0\right\} .
$$

Let $\pi: \mathbb{C}^{n+1} \ni\left(x_{1}, \ldots, x_{n+1}\right) \mapsto\left(x_{1}, \ldots, x_{n}\right) \in \mathbb{C}^{n}$ be the projection. Let $Y=\operatorname{Sing}\left(X_{1}\right)$ be the singular locus of $X_{1}$ and set $Y^{\prime}=\pi(Y)$. It is easy to see that $Y^{\prime}$ is contained in some hypersurface $H \subset\left\{x: x_{n+1}=0\right\}$. The hypersurface $H$ is described by a reduced polynomial $h \in \mathbb{C}\left[x_{1}, \ldots, x_{n}\right]$. Without restriction of generality we can assume that $\pi(x) \notin H$. Moreover, we can assume that $h(0) \neq 0$ and $0 \notin X_{1}$.

Now consider the $h$-process $F_{h}$ and $X_{2}:=\operatorname{cl}\left(F_{h}\left(X_{1}\right)\right)$. From Lemma 2.4 we see that $X_{1} \backslash V_{h} \cong X_{2} \backslash H_{h}$ and $0 \notin X_{2}$.

Consider the mapping

$$
\begin{aligned}
\sigma: \mathbb{C}^{n+1} \ni\left(x_{1}, \ldots, x_{n}, x_{n+1}\right) & \mapsto \\
\left(x_{1} x_{n+1}, x_{2} x_{n+1}, \ldots, x_{n} x_{n+1}, x_{n+1}\right) & \in \mathbb{C}^{n+1} .
\end{aligned}
$$

Since $h(0) \neq 0$ and $0 \notin X_{1}$, we have $\sigma^{-1}\left(X_{2}\right)=\sigma^{-1}\left(X_{2}\right) \backslash H_{h} \cong X_{1} \backslash V_{h} \cong$ $X \backslash \phi^{-1}\left(V_{h}\right)$. Hence, if we take $U:=\sigma^{-1}\left(X_{2}\right)$ and $f=\phi^{-1} \circ F_{h}^{-1} \circ \sigma$, then $f: U \rightarrow X$ is an open embedding and $U_{x}:=f(U)$ is a smooth affine neighborhood we are looking for.

COROllary 2.6. Let $X$ be a smooth $n$-dimensional algebraic variety. Then there is an open covering $\left\{U_{1}, \ldots, U_{k}\right\}$ of $X$ such that every $U_{i}$ is isomorphic to a closed hypersurface $S_{i} \subset \mathbb{C}^{n+1}$.

Corollary 2.7. Let $X$ be an $n$-dimensional algebraic variety $(n>0)$. Then there are infinitely many smooth affine hypersurfaces $Y_{s} \subset \mathbb{C}^{n+1}$, $s \in \mathbb{N}$, such that each $Y_{s}$ is birationally isomorphic to $X$, and $Y_{s}$ is not isomorphic to $Y_{s^{\prime}}$ for $s \neq s^{\prime}$.

Proof. We construct the sequence of hypersurfaces $Y_{s} \subset \mathbb{C}^{n}, s \in \mathbb{N}$ inductively. A hypersurface $Y_{1} \subset X$ exists by Corollary 2.6. Now assume that we have a sequence $Y_{k} \subset Y_{k-1} \subset \ldots \subset Y_{1}$ (with all inclusions strict) such that all $Y_{i}$ are isomorphic to hypersurfaces in $\mathbb{C}^{n+1}$. We show how to construct $Y_{k+1}$. Take points $a, b \in Y_{k}$ and let $Y_{k+1}$ be a Zariski open neighborhood $U_{a} \subset Y_{k} \backslash\{b\}$ of $a$ which is isomorphic to a smooth hypersurface in $\mathbb{C}^{n+1}$. Of course we have the strict inclusion $Y_{k+1} \subset Y_{k}$.

Now if $s \neq s^{\prime}$ then $Y_{s} \subset Y_{s^{\prime}}$ or conversely (and the inclusion is strict). We can assume that $Y_{s} \subset Y_{s^{\prime}}$. If $Y_{s}$ were isomorphic to $Y_{s^{\prime}}$, then we would 
have an injective mapping $f: Y_{s^{\prime}} \rightarrow Y_{s} \subset Y_{s^{\prime}}$. But by [4] this means that the mapping $f: Y_{s^{\prime}} \rightarrow Y_{s^{\prime}}$ is an isomorphism, in particular $Y_{s}=Y_{s^{\prime}}$, a contradiction.

We also have a stronger version of Theorem 2.5:

Theorem 2.8. Let $X_{1}, \ldots, X_{r}$ be $n$-dimensional algebraic varieties. Then there are Zariski open subsets $U_{i} \subset X_{i}$ such that every $U_{i}$ is isomorphic to a closed smooth hypersurface $S_{i} \subset \mathbb{C}^{n+1}$ and $S_{i} \cap S_{j}=\emptyset$ for $i \neq j$.

Proof. Let $\phi_{j}: X_{j} \rightarrow \mathbb{C}^{n+1}$ be birational embeddings as in Lemma 2.1. Define $X_{j}^{\prime}:=\phi\left(X_{j}\right)$. We can assume that $X_{i}^{\prime} \neq X_{j}^{\prime}$ for $i \neq j$. Let $X:=$ $\bigcup_{j=1}^{r} X_{j}$ and $X^{\prime}:=\bigcup_{j=1}^{r} X_{j}^{\prime}$.

Now we follow the proof of Theorem 2.5. By changing variables we can assume that

$$
X^{\prime}:=\left\{x \in \mathbb{C}^{n+1}: x_{n+1}^{k}+\sum_{j=1}^{k-1} a_{j}\left(x_{1}, \ldots, x_{n}\right) x_{n+1}^{k-j}=0\right\} .
$$

Let $\pi: \mathbb{C}^{n+1} \ni\left(x_{1}, \ldots, x_{n+1}\right) \mapsto\left(x_{1}, \ldots, x_{n}\right) \in \mathbb{C}^{n}$ be the projection. Let $Y=\operatorname{Sing}\left(X^{\prime}\right)$ and $Y^{\prime}=\pi(Y)$. Then $Y^{\prime}$ is contained in some hypersurface $H \subset\left\{x: x_{n+1}=0\right\}$, described by a reduced polynomial $h \in \mathbb{C}\left[x_{1}, \ldots, x_{n}\right]$. Without restriction of generality we can assume that $h(0) \neq 0$ and $0 \notin X^{\prime}$.

Now consider the $h$-process $F_{h}$ and set $X^{\prime \prime}:=\operatorname{cl}\left(F_{h}\left(X^{\prime}\right)\right)$. From Lemma 2.4 we see that $X^{\prime} \backslash V_{h} \cong X^{\prime \prime} \backslash H_{h}$ and $0 \notin X^{\prime \prime}$.

Consider the mapping $\sigma: \mathbb{C}^{n+1} \rightarrow \mathbb{C}^{n+1}$. Since $h(0) \neq 0$ and $0 \notin X^{\prime \prime}$, we have

$$
\sigma^{-1}\left(X^{\prime \prime}\right)=\sigma^{-1}\left(X^{\prime \prime}\right) \backslash H_{h} \cong X^{\prime} \backslash V_{h} \cong X \backslash \phi^{-1}\left(V_{h}\right) \cong \bigcup_{j=1}^{r}\left(X_{j} \backslash \phi_{j}^{-1}\left(V_{h}\right)\right) .
$$

Hence, it is enough to take $U_{j}:=X_{j} \backslash \phi_{j}^{-1}\left(V_{h}\right)$ and $S_{j}:=\sigma^{-1} \circ F_{h} \circ \phi\left(U_{j}\right)$.

3. Known results. Let us recall some facts about the set of points at which a polynomial mapping $f: \mathbb{C}^{n} \rightarrow \mathbb{C}^{m}$ is not proper (cf. [2], [3]).

Definition 3.1. Let $f: \mathbb{C}^{n} \rightarrow \mathbb{C}^{m}$ be a polynomial generically-finite mapping. We say that $f$ is proper at a point $y \in \mathbb{C}^{n}$ if there exists an open neighborhood $U$ of $y$ such that $\operatorname{res}_{f^{-1}(U)} f: f^{-1}(U) \rightarrow U$ is a proper map.

REMARK 3.2. A polynomial mapping $f$ is finite if and only if it is proper at every point $y \in \mathbb{C}^{m}$.

In [2], [3] we have studied the set $S_{f}$ of points at which a mapping $f: \mathbb{C}^{n} \rightarrow \mathbb{C}^{m}$ is not proper. To formulate the main result of this study 
we need the notion of a $\mathbb{C}$-uniruled variety. The following proposition was proved in [3], Proposition 5.1:

Proposition 3.3. Let $X$ be an irreducible affine variety of dimension $\geq 1$. The following conditions are equivalent:

(1) for every $x \in X$ there is an affine parametric line $\Gamma_{x}$ in $X$ through $x$;

(2) there exists a Zariski-open, non-empty subset $U$ of $X$ such that for every $x \in U$ there is an affine parametric line $\Gamma_{x}$ in $X$ through $x$;

(3) there exists a subset $U$ of $X$ of the second Baire category such that for every point $x \in U$ there is an affine parametric line $\Gamma_{x}$ in $X$ through $x$;

(4) there exists an affine variety $W$ with $\operatorname{dim} W=\operatorname{dim} X-1$ and a dominant polynomial mapping $\phi: W \times \mathbb{C} \rightarrow X$.

Now we can introduce our basic definition (cf. [3]):

Definition 3.4. An affine irreducible variety $X$ is called $\mathbb{C}$-uniruled if it is of dimension $\geq 1$ and satisfies one of the equivalent conditions (1)(4) of Proposition 3.3. More generally, if $X$ is an affine variety then $X$ is called $\mathbb{C}$-uniruled if it has pure dimension $\geq 1$ and every component of $X$ is $\mathbb{C}$-uniruled. Additionally we assume that the empty set is $\mathbb{C}$-uniruled.

Finally we have the following description of the set $S_{f}$ (cf. [3], Theorem 5.8):

Proposition 3.5. Let $f: \mathbb{C}^{n} \rightarrow \mathbb{C}^{m}$ be a polynomial generically-finite mapping. Then the set $S_{f}$ of points at which the mapping $f$ is not proper is either empty or it has pure dimension $n-1$. Moreover, the variety $S_{f}$ is $\mathbb{C}$-uniruled.

In what follows we also need the following theorem (cf. [3], Theorem 5.4):

THEOREM 3.6. Let $X$ be an affine variety and $Y \subset X$ be a closed subvariety. Let $f: Y \rightarrow \mathbb{C}^{n}$ be a polynomial mapping. Assume that $\operatorname{dim} X \leq n$. Then there exists a polynomial mapping $F: X \rightarrow \mathbb{C}^{n}$ such that

(1) $\operatorname{res}_{Y} F=f$,

(2) the mapping $\operatorname{res}_{X \backslash Y} F: X \backslash Y \rightarrow \mathbb{C}^{n}$ is quasi-finite.

4. A characterization of components of $S_{f}$. Now we can prove our second main result.

Theorem 4.1. Let $2 \leq n \leq m$ and let $X \subset \mathbb{C}^{m}$ be an $n$-1-dimensional $\mathbb{C}$-uniruled subset of $\mathbb{C}^{m}$. Then there is a polynomial quasi-finite mapping $F: \mathbb{C}^{n} \rightarrow \mathbb{C}^{m}$ such that $X \subset S_{F}$.

Proof. First assume that $F: \mathbb{C}^{n+1} \rightarrow \mathbb{C}^{n+1}$ and $X=S \times \mathbb{C}$ where $S \subset \mathbb{C}^{n}=\left\{x \in \mathbb{C}^{n+1}: x_{n+1}=0\right\}$. This means that the subset $X$ is 
described by a polynomial $h \in \mathbb{C}\left[x_{1}, \ldots, x_{n}\right]$. Consider the mapping

$$
\begin{aligned}
F: \mathbb{C}^{n+1} \ni\left(x_{1}, \ldots, x_{n}, x_{n+1}\right) & \mapsto \\
\left(x_{1}, \ldots, x_{n}, h\left(x_{1}, \ldots, x_{n}\right) x_{n+1}^{2}+x_{n+1}\right) & \in \mathbb{C}^{n+1} .
\end{aligned}
$$

It is easy to see that $F$ is a quasi-finite mapping and $S_{F}=X$.

We now turn to the general case. Let $X \subset \mathbb{C}^{m}$ be an $n$-1-dimensional $\mathbb{C}$-uniruled algebraic set. We have a decomposition $X=\bigcup_{j=1}^{r} X_{j}$, where $X_{j}$ are $n$-1-dimensional $\mathbb{C}$-uniruled irreducible varieties.

From Proposition 3.3, there are affine varieties $W_{j}$ with $\operatorname{dim} W_{j}=n-2$ and dominant polynomial mappings $\phi_{j}: W_{j} \times \mathbb{C} \rightarrow X_{j}$. By Corollary 2.7, we can assume that $W_{j} \subset \mathbb{C}^{n-1}$ and $W_{i} \cap W_{j}=\emptyset$ for $i \neq j$. Put $Y_{j}:=W_{j} \times \mathbb{C}$. Hence $Y_{j} \subset \mathbb{C}^{n}$ and $Y_{i} \cap Y_{j}=\emptyset$ for $i \neq j$.

By the first part of our proof there is a quasi-finite polynomial mapping $G: \mathbb{C}^{n} \rightarrow \mathbb{C}^{n}$ such that $S_{G}=\bigcup_{j=1}^{r} Y_{j}$. Since $G$ is quasi-finite, by the Zariski Main Theorem there is an affine variety $Z$ which contains $\mathbb{C}^{n}$ as an open dense subset and a finite mapping $G_{1}: Z \rightarrow \mathbb{C}^{n}$ such that res $\mathbb{C}^{n} G_{1}=G$. Set $P:=Z \backslash \mathbb{C}^{n}$. It is easy to see that $S_{G}=G_{1}(P)$. In particular if $P=\bigcup_{i=1}^{s} P_{i}$, then for every $Y_{j}$ there is an appropriate $P_{i}$ such that $Y_{j}=G_{1}\left(P_{i}\right)$.

Now we return to the set $X=\bigcup_{j=1}^{r} X_{j}$. Recall that we have dominant mappings $\phi_{j}: Y_{j} \rightarrow X_{j}$. Since $Y_{i} \cap Y_{j}=\emptyset$ for $i \neq j$, we also have the mapping $\phi=\bigcup_{i=1}^{r} \phi_{i}$. Consider the mapping $f: P \ni z \mapsto \phi \circ G_{1}(z) \in \mathbb{C}^{m}$. It is easy to see that $\operatorname{cl}(f(P))=X$. By Theorem 3.6 we can extend $f$ to a mapping $F_{1}: Z \rightarrow \mathbb{C}^{m}$ such that

(1) $\operatorname{res}_{P} F_{1}=f$,

(2) the mapping $\operatorname{res}_{Z \backslash P} F_{1}: Z \backslash P \rightarrow \mathbb{C}^{m}$ is quasi-finite.

If we set $F=\operatorname{res}_{\mathbb{C}^{n}} F_{1}$, then the mapping $F$ is quasi-finite and $X \subset S_{F}$ by the construction.

Corollary 4.2. Let $2 \leq n \leq m$ and let $X \subset \mathbb{C}^{m}$ be an irreducible variety. Then $X$ is an irreducible component of the set $S_{F}$ for some genericallyfinite polynomial mapping $F: \mathbb{C}^{n} \rightarrow \mathbb{C}^{m}$ if and only if $X$ is $\mathbb{C}$-uniruled and $\operatorname{dim} X=n-1$.

The author does not know whether the (last) inclusion in Theorem 4.1 can be replaced by equality. However in some cases it is possible:

Proposition 4.3. Let $2 \leq n \leq m$ and let $S_{1}, \ldots, S_{r} \subset \mathbb{C}^{m}$ be affine $n$-1-dimensional irreducible varieties such that there are finite mappings $\phi_{i}: \mathbb{C}^{n-1} \rightarrow S_{i}, i=1, \ldots, r$. Then there exists a polynomial mapping $F$ : $\mathbb{C}^{n} \rightarrow \mathbb{C}^{m}$ with finite fibers such that $S_{F}=\bigcup_{i=1}^{r} S_{i}$. 
Pr o of. Consider the mapping

$G: \mathbb{C}^{n} \ni\left(x_{1}, \ldots, x_{n}\right) \mapsto\left(x_{1}, \ldots, x_{n-1},\left(\prod_{i=1}^{r}\left(x_{1}-i\right)\right) x_{n}^{2}+x_{n}\right) \in \mathbb{C}^{n}$.

It is easy to see that $G$ is quasi-finite and $S_{G}=\left\{x \in \mathbb{C}^{n}: \prod_{i=1}^{r}\left(x_{1}-i\right)=0\right\}$. In particular $S_{G}=\bigcup_{i=1}^{r} W_{i}$, where $W_{i} \cong \mathbb{C}^{n-1}$ for $i=1, \ldots, r$ and $W_{i} \cap$ $W_{j}=\emptyset$ for $i \neq j$.

Now let $S_{1}, \ldots, S_{r} \subset \mathbb{C}^{m}$ be affine $n$-1-dimensional irreducible varieties such that there are finite mappings $\phi_{i}: \mathbb{C}^{n-1} \rightarrow S_{i}, i=1, \ldots, r$. We can assume that $\phi_{i}: W_{i} \rightarrow S_{i}$. In particular we have a finite mapping $\phi$ : $\bigcup_{i=1}^{r} W_{i} \rightarrow \bigcup_{i=1}^{r} S_{i} \subset \mathbb{C}^{m}$. By [2], Proposition 21, we can extend $\phi$ to a finite mapping $\Phi: \mathbb{C}^{n} \rightarrow \mathbb{C}^{m}$. Now it is enough to set $F=\Phi \circ G$.

Corollary 4.4. Let $2 \leq n \leq m$ and let $S_{1}, \ldots, S_{r} \subset \mathbb{C}^{m}$ be $n-1$ dimensional linear subspaces. Then there exists a polynomial mapping $F$ : $\mathbb{C}^{n} \rightarrow \mathbb{C}^{m}$ with finite fibers such that $S_{F}=\bigcup_{i=1}^{r} S_{i}$.

\section{References}

[1] R. Hartshorne, Algebraic Geometry, Springer, New York, 1987.

[2] Z. Jelonek, The set of points at which a polynomial map is not proper, Ann. Polon. Math. 58 (1993), 259-266.

[3] - , Testing sets for properness of polynomial mappings, Math. Ann. 315 (1999), $1-35$.

[4] K. Nowak, Injective endomorphisms of algebraic varieties, ibid. 299 (1994), 769-778.

Institute of Mathematics

Polish Academy of Sciences

Św. Tomasza 30

31-027 Kraków, Poland
Institute of Mathematics Jagiellonian University Reymonta 4 30-059 Kraków, Poland E-mail: jelonek@im.uj.edu.pl

Reçu par la Rédaction le 25.6.1999

Révisé le 5.2.2000 et le 16.10.2000 